\section{Targeting Wnt in pulmonary fibrosis}

\section{By Tim Fulmer, Senior Writer}

German researchers have identified WISP1-a component of the Wnt signaling pathway-as a potential therapeutic target in pulmonary fibrosis. ${ }^{1}$ Talks are already underway between the researchers and an undisclosed company to develop WISP1 inhibitors for the disease.

An improperly regulated wound-healing response to injury or infection can lead to fibrosis in solid organs. In the lung, fibrosis is caused by the persistent irritation of inhaled toxins or, in cases of idiopathic pulmonary fibrosis (IPF), by unknown stimuli. In both cases, lung architecture and function deteriorate significantly over time, eventually leading to respiratory failure and death in the absence of a transplant. ${ }^{2}$

Although previous research had shown that Wnt signaling is upregulated in pulmonary fibrosis, ${ }^{3}$ cardiac fibrosis ${ }^{4}$ and muscle fibrosis, ${ }^{5}$ the challenge has been finding a molecular target in the pathway that can alleviate or slow disease progression.

Oliver Eickelberg of Ludwig Maximilian University of Munich and colleagues set about the target search by looking at the gene expression profiles of alveolar epithelial type II cells isolated from mouse models of chemically induced pulmonary fibrosis. Alveolar epithelial cells play a key role in IPF.

The mRNAs of multiple Wnt signaling pathway components were significantly upregulated in the fibrotic mice compared with that seen in healthy, saline-treated controls $(p<0.02)$. The group homed in on one of the upregulated components, the connective tissue growth factor WNT1 inducible signaling pathway protein 1 (WISP1), because other groups had already correlated abnormal expression of the protein with lung fibrosis ${ }^{6}$ and lung cancer. ${ }^{7}$

The new work developed a strategy for locally blocking WISP1 to treat lung fibrosis.

In cultured alveolar epithelial cells from fibrotic mouse lungs, targeting Wisp1 with antibodies or small interfering RNA significantly decreased cellular proliferation compared with that seen using control IgG or control scrambled peptide ( $p<0.02$ for both).

Finally, the researchers tested whether blocking proliferation of alveolar epithelial cells in vivo could have a disease-modifying effect. Indeed, in mouse models of chemically induced lung fibrosis, delivery of Wisp1-neutralizing antibodies directly to lung tissue via the trachea lowered pulmonary fibrosis, as evidenced by a significant decrease in collagen deposition compared with that seen using control antibody $(p<0.02)$.
The results were reported in the Journal of Clinical Investigation. Eickelberg is chairman of experimental pneumology in the Comprehensive Pneumology Center at the university and director of the Institute of Lung Biology and Disease at the Helmholtz Center Munich.

\section{Breathing easier}

Eickelberg told SciBX that he is in talks with an undisclosed company to design and develop a human mAb targeting WISP1.

According to Eickelberg, the first hurdle to cross will be delivery. "A key step towards the clinic is designing a formulation that allows inhaled delivery of the WISP1-targeting therapeutic antibodies," he said. "The advantage of inhalation is that it can locally modulate Wnt signaling in the fibrotic lung without the side effects potentially associated with systemic delivery of a Wnt pathway inhibitor."

But Shelia Violette, VP of research at fibrosis-focused Stromedix Inc., wants to see more research on how the anti-WISP1 antibodies work. "It's unclear what WISP1 interactions the paper's neutralizing antibodies are interfering with to elicit the antifibrotic effect in vivo," she said. "To better validate WISP1 as a target in fibrosis, it would be useful to determine if the antibodies are blocking a specific interaction or potentially multiple interactions in fibrosis."

Those additional studies could also include targeting WISP1 in models of progressive fibrotic disease, said David Liu, VP of research at FibroGen Inc.

"The paper's bleomycin model of chemically induced lung fibrosis is clinically relevant and a standard model of lung fibrosis," he said. "However, it does not address disease progression, because the antiWISP1 antibodies are given together with the chemical irritant, not after fibrosis is established. Thus, based on that model, it's not clear whether targeting WISP1 can prevent or reverse fibrotic progression."

Violette also wanted to know "if there are any potential safety issues related to chronic blockade of WISP1 activity, as most fibrotic disease would involve chronic therapy."

Clinical development of antifibrotics also comes with a host of challenges, noted Stromedix cofounder and CEO Michael Gilman. "Fibrotic diseases develop slowly over the course of decades, which raises the problem of how to run an informative trial in a reasonable period of time. A typical first-in-patient trial is unlikely to run more than three months," he said.

Thus, according to Gilman, the challenge is "choosing a measurable endpoint that reflects a tissue-level phenomenon like fibrosis. Readouts of organ function are indirect and potentially deceptive. Ideally one will be able to build a strong case for the antifibrotic activity of a compound based on biomarker measurements in the affected tissue."

Despite the long duration of many fibrotic diseases, Gilman told SciBX that some investigators believe that by the time a patient actually presents with symptoms "their condition is too advanced to be affected by an antifibrotic intervention." 


\section{TARGETS \& MECHANISMS}

Stromedix's lead compound, STX-100, is in preclinical development for lung fibrosis. The company's approach "will incorporate the evaluation of biomarkers as early markers of proof of mechanism," Violette said.

The humanized anti-integrin $\alpha_{\mathrm{v}} \beta_{6} \mathrm{mAb}$, which the company in-licensed from Biogen Idec Inc., is due to start a Phase IIa trial in chronic allograft nephropathy by mid-year.

FibroGen's FG-3019, a human mAb against connective tissue growth factor (CTGF), decreased fibrosis and increased organ function in various preclinical models of fibrotic disorders and was also well tolerated in a single-dose Phase I safety trial in IPF patients, according to Liu. The company is planning Phase II trials of FG-3019 in multiple indications, including IPF, he said.

Eickelberg did not disclose the patent and licensing status of the findings reported in the JCI article.

Fulmer, T. SciBX 2(13); doi:10.1038/scibx.2009.520

Published online April 2, 2009

\section{REFERENCES}

1. Konigshoff, M. et al. J. Clin. Invest.; published online March 16, 2009; doi:10.1172/JCl33950

Contact: Oliver Eickelberg, Ludwig Maximilian University of Munich, Munich, Germany

e-mail: oliver.eickelberg@helmholtz-muenchen.de

2. Wilson, M. \& Wynn, T. Mucosal Immunol. 2, 103-121 (2009)

3. Konigshoff, M. et al. PLOS ONE 3, e2142; published online May 14, 2008; doi:10.1371/journal.pone.0002142

4. Colston, J. et al. Am. J. Physiol. Heart Circ. Physiol. 293, H1839-H1846 (2007)

5. Brack, A. et al. Science 317, 807-810 (2007)

6. Selman, M. et al. Am. J. Respir. Crit. Care Med. 173, 188-198 (2006)

7. Chen, P.-P. et al. PLoS ONE 2, e534; published online June 20, 2007; doi:10.1371/journal.pone.0000534

COMPANIES AND INSTITUTIONS MENTIONED

Biogen Idec Inc. (NASDAQ:BIIB), Cambridge, Mass.

FibroGen Inc., San Francisco, Calif.

Helmholtz Center Munich, Munich, Germany

Ludwig Maximilian University of Munich, Munich, Germany

Stromedix Inc., Cambridge, Mass. 